\title{
Spatio-temporal imaging of the hemoglobin in the compressed breast with diffuse optical tomography
}

\author{
Gregory Boverman ${ }^{1}$, Qianqian Fang ${ }^{2}$, Stefan A Carp ${ }^{2}$, Eric L Miller ${ }^{3}$, \\ Dana H Brooks ${ }^{4}$, Juliette Selb ${ }^{2}$, Richard H Moore ${ }^{5}$, Daniel B Kopans ${ }^{5}$ \\ and David A Boas ${ }^{2}$ \\ ${ }^{1}$ Biomedical Engineering Department, Rensselaer Polytechnic Institute, Jonsson Engineering \\ Center, Troy, NY 12180, USA \\ 2 Martinos Center for Biomedical Imaging, Massachusetts General Hospital, Charlestown, \\ MA 02129, USA \\ ${ }^{3}$ Department of Electrical and Computer Engineering, Tufts University, Medford, MA 02153, \\ USA \\ ${ }^{4}$ Department of Electrical and Computer Engineering, Northeastern University, Boston, \\ MA 02115, USA \\ 5 Breast Imaging Center, Massachusetts General Hospital, 15 Parkman St, Boston, MA 02114, \\ USA \\ E-mail: boverg@rpi.edu
}

Received 15 September 2006, in final form 2 April 2007

Published 23 May 2007

Online at stacks.iop.org/PMB/52/3619

\begin{abstract}
We develop algorithms for imaging the time-varying optical absorption within the breast given diffuse optical tomographic data collected over a time span that is long compared to the dynamics of the medium. Multispectral measurements allow for the determination of the time-varying total hemoglobin concentration and of oxygen saturation. To facilitate the image reconstruction, we decompose the hemodynamics in time into a linear combination of spatiotemporal basis functions, the coefficients of which are estimated using all of the data simultaneously, making use of a Newton-based nonlinear optimization algorithm. The solution of the extremely large least-squares problem which arises in computing the Newton update is obtained iteratively using the LSQR algorithm. A Laplacian spatial regularization operator is applied, and, in addition, we make use of temporal regularization which tends to encourage similarity between the images of the spatio-temporal coefficients. Results are shown for an extensive simulation, in which we are able to image and quantify localized changes in both total hemoglobin concentration and oxygen saturation. Finally, a breast compression study has been performed for a normal breast cancer screening subject, using an instrument which allows for highly accurate co-registration of multispectral diffuse optical measurements with an x-ray tomosynthesis image of the breast. We are able to quantify the global return of blood to the breast following compression, and, in addition,
\end{abstract}


localized changes are observed which correspond to the glandular region of the breast.

(Some figures in this article are in colour only in the electronic version)

\section{Introduction}

Diffuse optical tomography (DOT) has shown promise as an adjunct to x-ray mammography (McBride et al 1999, Franceschini et al 1997, Pogue et al 2001, Shah et al 2001, Cerussi et al 2001, Li et al 2003), in particular because it allows us to image the concentration of blood and a number of other physiologically significant chromophores within the breast, albeit with a considerably lower level of spatial resolution than possible with mammography. Multiwavelength measurements also allow determination of the spatial distribution of blood oxygen saturation $\left(\mathrm{SO}_{2}\right)$. Tumors appear to differ from normal tissue in total hemoglobin concentration (HbT) (Fishkin et al 1997), and may further differ from normal tissue in $\mathrm{SO}_{2}$ (Dehghani et al 2003) and in their scattering properties (Srinivasan et al 2005). Research has also shown a correlation between tumor oxygen saturation and likelihood to metastasize (Brizel et al 1996). Moreover, DOT can achieve a relatively high temporal resolution, allowing the imaging of hemodynamics in both space and time.

In this paper, we specifically focus on the problem of imaging the hemodynamic response of the breast to compression. This work is motivated by our efforts to develop a combined x-ray-DOT mammography instrument (Zhang et al 2005, Li et al 2003). In our clinical experiments, we make use of an optical imaging system which has been incorporated into an X-ray tomosynthesis machine (Niklason et al 1997). We are able to acquire both images without changing the position of the breast, allowing for highly accurate co-registration of the optical and three-dimensional $\mathrm{x}$-ray images. We have observed that compression induces rapid hemodynamic changes in the breast, and it is the DOT imaging of the time-varying mammographically-compressed breast that is the subject of this paper. Recently published reports have studied the bulk hemodynamic response of the breast (Carp et al 2006) to compression, showing a tendency of blood to return to the breast during pressure-relaxation of the tissue following the initial compression. In that study, changes in oxygenation were observed which allowed the determination of the level of oxygen consumption of normal breast tissue using diffuse optical measurements. Compression-induced differences in oxygen consumption between tumor and normal tissue, as noted above, may indeed prove to be a valuable source of imaging contrast. Here we extend this bulk tissue study to the imaging of hemodynamics, and observe the spatial distribution of the blood return to the breast. In addition, we have also seen localized, relatively large changes in oxygen saturation, which, we hypothesize, are due to temporary hypoxia of regions within the breast in which blood flow and concentration have been reduced due to compression.

There have been several recent reports on methods for dynamic DOT imaging. Barbour et al (2001) introduced the idea of imaging a dynamic medium, using diffuse optical measurements. Collaborators subsequently published an analysis of the performance of linear dynamic imaging algorithms for a number of data types (Graber et al 2002). Members of the same research group proceeded to apply dynamic imaging methods to optical imaging of the brain, in response to breath-holding (Bluestone et al 2001). Hebden et al (2004) reported on dynamic imaging of the infant brain, using a linear perturbational approach and differenceimaging. Barbour et al (2003) have also applied dynamic imaging methods to the imaging of 
the breast, showing that the global spatio-temporal properties of a breast containing a tumor may differ from those of normal tissue. Recently, Intes et al (2003) published a study making use of optical dynamic imaging with a contrast agent, indocyanine green (ICG). This report investigated the kinetics of ICG uptake and washout in subjects with benign and malignant lesions. In a subject with an invasive ductal carcinoma, the tumor tissue exhibited slower uptake and washout rates than normal tissue. In our work, we make use of breast compression, which is already a component of x-ray mammographic screening, as a form of contrast, imaging low-frequency dynamics of total hemoglobin concentration and oxygen saturation following a sharp compression of the breast.

A number of modeling approaches have been proposed for the solution of the spacetime image-reconstruction problem. The application of the extended Kalman filter (EKF) to optical tomography was introduced in Eppstein et al (1999). More recently, the EKF approach was applied to the spatio-temporal imaging problem (Prince et al 2003, Kolehmainen et al 2003), in order to introduce temporal smoothing. A recent study also examined the performance of Kalman filtering with DOT using physiological a priori information (Diamond et al 2006). In the case where there is a priori information about the shape of a given voxel's time course, for example in functional brain imaging, where the general shape of the hemodynamic response function (HRF) has been demonstrated to have a particular pattern, spatio-temporal basis functions (Zhang et al 2005) have been utilized in order to exploit this prior information. A recent report compared a number of spatio-temporal regularization approaches, including Kalman filtering and spatio-temporal regularization, placing them in a common statistical framework (Zhang et al 2005).

While the Kalman filter and extended Kalman filter (EKF) do possess the advantage of providing a recursive solution of the spatio-temporal estimation problem, for realistic, threedimensional problems, these approaches still involve a degree of computational complexity, as the update step, in which the Kalman gain is computed, generally requires the inversion of a large matrix. For the clinical measurement given in this paper, for example, computing the Kalman gain for each time instant would require the inversion of a matrix of size $14378 \times 14378$. In this work, we do not assume that a real-time solution of the dynamic estimation problem is necessary, and thus we estimate all of the unknowns from all of the data simultaneously, using a regularized inversion approach, in an analogous manner to methods applied to static image reconstruction. This methodology introduces a great deal of computational complexity as well, but we make use of iterative methods, which avoid explicit matrix inversion, to lessen the computational demand.

In our work, since we do not have good prior knowledge of the shape of a given voxel's time course, we instead use temporal basis functions which enforce a degree of smoothness in time. Specifically, we implement cubic-spline basis functions (Parker et al 1983). Moreover, the fact that this basis consists of time-shifted versions of the same kernel function allows us to easily introduce explicit spatio-temporal regularization (Zhang et al 2005, Brooks et al 1999), penalizing the dissimilarity between the images of spatio-temporal coefficients which are adjacent in time, thus imposing a further degree of temporal smoothness. An attractive feature of the use of a single cost function in the dynamic estimation is that we can monitor the cost to assess the convergence of our optimization algorithm. It also provides a useful criterion for the tuning of spatial and temporal regularization parameters.

Our interest in the fully nonlinear reconstruction of the space and time-varying nature of the medium, coupled with a degree of practical experience we have gained through the analysis of clinical data, have led us to posit in this paper a very specific method for decomposing the spacetime properties of the breast. Specifically, we were inspired by the commonly encountered temporally static reconstruction problem in which only the spatial structure is 
to be recovered, where one commonly decomposes the absorption into two components: a bulk background term, typically assumed to be spatially constant, along with a perturbational component that accounts for spatial variations. While the latter term is typically assumed to be 'small' in some sense when linear reconstruction methods are employed, the above decomposition is perfectly valid and often employed either explicitly or implicitly for nonlinear inversion approaches as well, where the 'smallness' assumption is not required.

Here we generalize this idea to the case of dynamic imaging. As discussed in detail in section 2.2, we decompose the spacetime variations in the absorption properties into two components: one that is temporally constant but may be spatially varying and a second that carries the temporal dynamics. Each of these in turn is further decomposed into a term that is spatially constant (like the bulk absorption mentioned previously) and one that does in fact vary as a function of space. This approach to parameterize the absorption was motivated by our experience with the processing of clinical data, where we have found it necessary to employ substantively different approaches to recover the static behavior of the absorption (where absolute imaging must be employed) in contrast to the dynamic behavior (where difference imaging can be used). In both cases, much as in the purely static reconstruction problem, it is crucial to capture the bulk properties in order to accurately recover the space-varying structure as well.

The estimation of the spatio-temporal dynamics is accomplished with a Newtonbased iterative image-reconstruction algorithm, which estimates all of the spatio-temporal coefficients from all of measurements simultaneously, using a combination of spatial regularization and temporal regularization. In order to solve the very large least-squares system which computes the search direction for the Newton update, we make use of LSQR (Paige and Saunders 1982), which is an iterative algorithm that only requires matrix-vector multiplies. An important advantage of iterative methods is that, for many realistic problems, convergence can be very rapid, requiring many fewer iterations than either $M$ or $N$ for an $M \times N$ matrix, in the case of a reasonable level of regularization. In general, we have found in our simulation experiments and in our analysis of clinical measurements that roughly $M / 10$ iterations are required to meet the convergence criterion for a typical level of regularization. Direct matrix inversion would be computationally prohibitive given the size of our imagereconstruction problem. In the case of our clinical measurements, we would need to invert a matrix of dimension $129402 \times 129402$ to solve the full spatio-temporal image-reconstruction problem.

To test our methods, we have conducted an extensive simulation study, with a breast geometry from a normal volunteer. After validating our algorithms and implementation with the simulation study, we proceeded to image a screening patient whose mammogram did not present any abnormalities. In both the simulation study and in our clinical experiments, we make measurements at two wavelengths, reconstructing the spatio-temporal absorption at each wavelength separately. Assuming reasonable, and spatially homogeneous, lipid and water concentrations, we then recovered the spatial distributions of oxy-hemoglobin ( $\mathrm{HbO})$ and deoxy-hemoglobin (HbR) concentrations, computing these quantities for each voxel by multiplying the estimated absorptions by a matrix derived from the extinction coefficients of these two chromophores (Li et al 2005, McBride et al 1999).

Computational considerations did not allow us to directly reconstruct the concentrations of $\mathrm{HbO}$ and $\mathrm{HbR}$ simultaneously while making use of the data at both wavelengths. The spatio-temporal reconstruction for one wavelength alone consumes roughly 9 GB of memory for a realistic three-dimensional breast geometry. However, in future we plan to make use of direct spectroscopic image-reconstruction methods (Li et al 2004, 2005, Srinivasan et al 2005, Zhang et al 2005), with a potentially great improvement in achievable image quality. 
We simulated hemodynamics in the breast with a nonlinear finite-difference-based forward model. In this simulation, measurements were made at 680 and $830 \mathrm{~nm}$ for a realistic experimental configuration, and with an inhomogeneous baseline $\mathrm{HbT}$ (total hemoglobin concentration, where $\mathrm{HbT}=\mathrm{HbO}+\mathrm{HbR}$ ) and $\mathrm{SO}_{2}$, where $\mathrm{SO}_{2}$ is defined as the ratio of $\mathrm{HbO}$ and $\mathrm{HbT}$. Also, the dynamics of $\mathrm{HbT}$ and $\mathrm{SO}_{2}$ exhibited a complex spatial structure, with distinct spatio-temporal behavior. We found that, even with a reasonable level of noise added to the measurements, we were able to accurately estimate the baseline images, the spatially homogeneous temporal variation, and the spatio-temporal behavior of both $\mathrm{HbT}$ and $\mathrm{SO}_{2}$. We further show that if there is significant variation in absorption over the imaging interval, it is important to take into account the temporal variation in absorption when estimating the baseline absorption.

Our preliminary clinical results show the time-courses for oxy-hemoglobin and the deoxyhemoglobin after a rapid compression of the breast, generally indicating a pressure-relaxationinduced return of oxygenated blood to the breast. In addition, we see localized changes in $\mathrm{HbT}$ and in $\mathrm{SO}_{2}$ which differ from the bulk temporal variation, and which correspond well to the glandular region of the breast.

Computationally, both our simulation and clinical studies required approximately $12 \mathrm{~h}$ of computation on an AMD Opteron workstation with 16 GB of memory. Much of the computation time involved the line search step of our Newton-based iterative optimization procedure, and we believe that a less accurate line search may result in little difference in the ultimate solution achieved with a great difference in computational cost. Also, the computation time could be considerably reduced by making use of parallel processing methods.

The organization of this paper is as follows: first, we discuss the forward model for DOT and describe our model for the reconstructed image. Next, we describe the cost functions which are used in the image reconstruction and detail the estimation of the spatiotemporal coefficients. We then show results for a fairly complex simulation example, involving measurements at two wavelengths, where we have assumed spatio-temporal variation in both oxygen saturation and total hemoglobin concentration. Finally, clinical results demonstrate the response of the breast of a normal screening subject to compression, as a function of both space and time.

\section{Computational and experimental methods}

\subsection{Sensor and experimental configuration}

In our clinical protocol, we place the breast under a standard level of mammographic compression and acquire optical data for approximately $45 \mathrm{~s}$, following which the optical probe is removed. Without changing the position of the breast, an x-ray tomosynthesis image of the compressed breast is then taken. The same level of compression is used for both the optical imaging and for mammographic imaging with the tomosynthesis instrument. During the optical imaging, CW (Siegel et al 1999) and RF (radio-frequency, $70 \mathrm{MHz}$ ) (Zhang et al 2005) images of the breast are taken simultaneously. Our RF system, as presently configured, does not have the temporal resolution for dynamic imaging, and is thus used solely to estimate the bulk absorption and scattering within the breast in this study.

The continuous-wave imaging system has two source wavelengths, $685 \mathrm{~nm}$ and $830 \mathrm{~nm}$, split evenly between 20 frequency-division multiplexed sources (i.e. 20 lasers at each wavelength), and 20 detector channels. Each laser is modulated at a different frequency, from $6.4 \mathrm{kHz}$ to $12.6 \mathrm{kHz}$, with an interval of $200 \mathrm{~Hz}$ between adjacent frequencies. All detector channels collect light from all sources simultaneously. Each detector channel consists of 


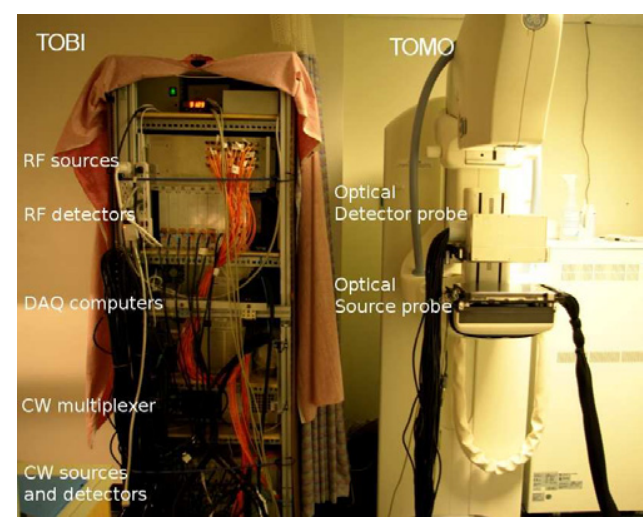

(a)

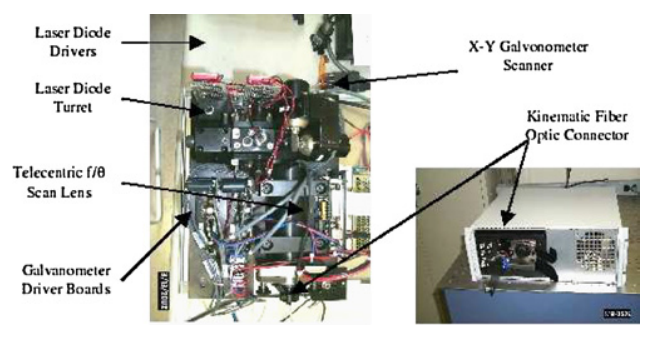

(b)

Figure 1. Imaging hardware used in our experiments: (a) TOBI (tomographic optical breast imaging) system, as it is used in conjunction with a 3D tomosynthesis mammography instrument. (b) Structure of the optical multiplexer.

a Hamamatsu APD module (C5460-01) followed by signal conditioning and amplification stages. Each photodetector preamp output is first highpass filtered to remove low-frequency signals from stable interference sources such as sunlight and $1 / f$ (flicker) noise generated by the electronics. The medium frequency components produced by fluorescent lamps at $120 \mathrm{~Hz}$ and its harmonics are somewhat attenuated as well. The lowpass filter following the gain control stage is for preventing aliasing, typically done as part of Nyquist sampling. Each detector channel is then sampled at $41.7 \mathrm{kHz}$ using a pair of National Instruments NI6052E data acquisition cards. In addition, we use a custom-built optical multiplexer to simultaneously make measurements at 685 and $830 \mathrm{~nm}$ (with both wavelengths encoded at a different frequency in the range from $6.4 \mathrm{kHz}$ to $12.6 \mathrm{kHz}$ ) at up to 150 different source positions. This multiplexer has the potential of switching between positions extremely rapidly, with transitions occurring in less than $1 \mathrm{~ms}$. In our experiments, we cycle through 28 source positions, chosen to maximize our coverage of the breast, seven times, with a dwell time of $200 \mathrm{~ms}$ for each position. The integration time at each source position is $100 \mathrm{~ms}$. Our TOBI (tomographic optical breast imaging) system, as it is used in conjunction with a tomosynthesis instrument, is shown in figure 1(a). The structure of the optical multiplexer is shown in figure 1(b).

\subsection{Forward model, cost functions and sensitivities}

In what follows, we describe the forward model for DOT, the cost functions used in imaging the spatio-temporal absorption distribution, the spatial and temporal regularization schemes employed, as well as the decomposition of the temporal response into temporal basis functions. Finally, we describe the optimization procedure which we have developed to solve the fully nonlinear spatio-temporal estimation problem.

For media in which $\mu_{s}$, the optical scattering coefficient, is much greater than $\mu_{a}$, the optical absorption coefficient, it is well known that the diffusion approximation, a secondorder partial differential equation, can well approximate radiative transport. In this paper, we 
make use of continuous-wave (CW) optical imaging, and, in this case, the diffusion equation can be written as

$$
-\nabla \cdot D(\mathbf{r}) \nabla \phi(\mathbf{r})+\mu_{a}(\mathbf{r}) \phi(\mathbf{r})=S_{0}(\mathbf{r})
$$

where the diffusion coefficient, $D$, is defined to be $\frac{1}{3(1-g) \mu_{s}}, g$ being the average cosine of the scattering angle. The source function is $S_{0}(\mathbf{r})$, and the photon fluence is given by $\phi(\mathbf{r})$. At the air-tissue boundary, we make use of a zero partial-flux boundary condition (Haskell et al 1994):

$$
\frac{1}{2} R_{\phi} \phi(\mathbf{r})-D(\mathbf{r}) R_{j} \hat{n} \cdot \nabla \phi(\mathbf{r})=0
$$

where $R_{\phi}$ and $R_{j}$ are the Fresnel reflection coefficients for the photon density and current, respectively, and $\hat{n}$ is the direction normal to the boundary.

We allow the absorption coefficient to be time-varying, and also take into account that each source is activated at a different point in time but our methods can easily be extended to the case of frequency-domain measurements. Thus we have a diffusion equation where the absorption coefficient is a function of both space and time:

$$
-\nabla \cdot D(\mathbf{r}) \nabla \phi(\mathbf{r}, t)+\mu_{a}(\mathbf{r}, t) \phi(\mathbf{r}, t)=S_{0}(\mathbf{r}, t) .
$$

In this work, we assume that the time scale over which $\mu_{a}$ varies is much longer than the time scale over which the CW diffusion equation is valid, and that, thus, the absorption can be assumed to be constant over the $100 \mathrm{~ms}$ during which each measurement is made. It is possible that the scattering coefficient may vary over time as well, but in this paper we are modeling and reconstructing changes in absorption only. We posit the following model for the time-varying absorption:

$$
\mu_{a}(\mathbf{r}, t)=\sqrt{a(\mathbf{r}, t)^{2}+\epsilon^{2}}
$$

where

$$
a(\mathbf{r}, t)=s(\mathbf{r})+v(\mathbf{r}, t) .
$$

The particular form of equation (4) is used to ensure that the absorption is always positive and that it is also always greater than $\epsilon=0.0035 \mathrm{~cm}^{-1}$.

Thus, we assume that the absorption can be broken into a static component, $s(\mathbf{r})$, and a component which varies in both space and time, $v(\mathbf{r}, t)$. This choice of model is based on observations of clinical measurements in which the optical signal tends not to vary more than $15 \%$ from its initial value. We further decompose $s(\mathbf{r})$ and $v(\mathbf{r}, t)$ into spatially constant and spatially-varying components:

$$
\begin{aligned}
& s(\mathbf{r})=\mu_{a}^{0}+p(\mathbf{r}) \\
& v(\mathbf{r}, t)=\sum_{l=1}^{L} c_{l} b_{l}(t)+\sum_{l=1}^{L} e_{l}(\mathbf{r}) b_{l}(t)
\end{aligned}
$$

where $\left\{b_{1}(t), b_{2}(t), \ldots, b_{L}(t)\right\}$ is a collection of $L$ temporal basis functions. Thus, we decompose the time-varying absorption into spatially and temporally constant, temporally varying and spatially constant, spatially varying and temporally constant and spatio-temporally varying components, denoted by the $\mu_{a}^{0}, p(\mathbf{r}), \sum_{l=1}^{L} c_{l} b_{l}(t)$ and $\sum_{l=1}^{L} e_{l}(\mathbf{r}) b_{l}(t)$, respectively. In fact, the decomposition into these components is somewhat redundant, as $\sum_{l=1}^{L} e_{l}(\mathbf{r}) b_{l}(t)$ includes $\sum_{l=1}^{L} c_{l} b_{l}(t)$ as a special case, but, in our optimization procedure, we first estimate the bulk temporal variation, described by the latter term, which is used as an initial guess for the former model, which describes the full spatio-temporal absorption variation. We assume 
that the static bulk absorption, $\mu_{a}^{0}$, is known a priori. The temporal basis functions are used in order to ensure a certain degree of temporal smoothness and because solving for the timevarying absorption at each point in time that a measurement is made (in our system, every $200 \mathrm{~ms}$ ) would be computationally prohibitive, given that we are solving the full nonlinear optimization problem for absorption for a complex three-dimensional medium.

Next, we consider the optimization of $a(\mathbf{r}, t)$ given a set of diffuse optical measurements. In what follows, we assume that the time scale of the changes in absorption is such that the absorption can be well approximated as being constant during the integration time (100 ms) of each measurement. In estimating $a(\mathbf{r}, t)$, we make use of a nonlinear optimization procedure. However, as the terms of $a(\mathbf{r}, t)$ are qualitatively different, we make use of two different cost functions. In doing so, we are driven by realistic experimental considerations related to DOT in practice. In order to estimate the 'static' (i.e. not varying with time) portion of $a(\mathbf{r}, t), s(\mathbf{r})$, we make use of an absolute cost function, while, in order to estimate the dynamics, we make use of difference-imaging. We have found that difference imaging is essential in estimating $v(\mathbf{r}, t)$ from experimental data, as an absolute cost function tends to compound systematic errors due to model mismatch, while difference imaging tends to cancel these errors. The absolute cost function used is

$$
C_{\text {absolute }}=\sum_{m=1}^{M} \sum_{n=1}^{N} \sum_{q=1}^{Q} \frac{\left(y_{m, n}^{q}-h_{m, n}^{q}\left(t_{m}^{q}, \mathbf{p}, \mathbf{c}, \mathbf{e}\right)\right)^{2}}{\left(\sigma_{m, n}^{q}\right)^{2}}
$$

where $y_{m, n}^{q}$ is the $q$ th repetition of a measurement due to source $m$ measured at detector $n$ and $\mathbf{p}=p(\mathbf{r}), t_{m}^{q}$ is the time at which this source is activated, $h_{m, n}^{q}\left(t_{m}^{q}, \mathbf{p}, \mathbf{c}, \mathbf{e}\right)$ is the hypothesized amplitude produced by our forward model, $\mathbf{c}=\left[c_{0} c_{1} \cdots c_{L}\right]^{T}, \mathbf{e}=\left[e_{0}(\mathbf{r}) e_{1}(\mathbf{r}) \cdots e_{L}(\mathbf{r})\right]^{T}$, and $\sigma_{m, n}^{q}$ is the standard deviation of $y_{m, n}^{q}$. For clinical data, we have found that, under compression, there are sufficient differences between our measurements and our model, even after calibration with a homogeneous phantom, that additional calibration parameters, one for each source and detector, must be introduced into the reconstruction (Boas et al 2001, Oh et al 2002). Thus, we make use of the following cost function:

$$
C_{\text {absolute,calibrated }}=\sum_{m=1}^{M} \sum_{n=1}^{N} \sum_{q=1}^{Q} \frac{\left(y_{m, n}^{q}-s_{m} d_{n} h_{m, n}^{q}\left(t_{m}^{q}, \mathbf{p}, \mathbf{c}, \mathbf{e}\right)\right)^{2}}{\left(\sigma_{m, n}^{q}\right)^{2}}
$$

where $s_{m}$ and $d_{n}$ are source and detector amplitude calibration coefficients, respectively. In contrast, the following cost function is used for difference-imaging:

$$
C_{\text {relative }}=\sum_{m=1}^{M} \sum_{n=1}^{N} \sum_{q=2}^{Q}\left[\frac{y_{m, n}^{q}}{y_{m, n}^{1}}-\frac{h_{m, n}^{q}\left(t_{m}^{q}, \mathbf{p}, \mathbf{c}, \mathbf{e}\right)}{h_{m, n}^{1}\left(t_{m}^{1}, \mathbf{p}, \mathbf{c}, \mathbf{e}\right)}\right]^{2} .
$$

In this we only try to match the ratio between the amplitude of the $q$ th repetition of the measurement for a particular source/detector pair and the amplitude recorded the first time this measurement was made.

We next concern ourselves with the problem of computing the Fréchet derivative of the forward model with respect to small perturbations in $e_{i}(\mathbf{r})$ and $p(\mathbf{r})$, where $e_{i}(\mathbf{r})$ is the spacevarying coefficient of the $i$ th temporal basis function and $p(\mathbf{r})$ is the static absorption image. These Fréchet derivatives are used to compute the Jacobian of the measurements with respect to our parameters, which is at the heart of our Newton-based estimation procedure. The sensitivity of a measurement due to a spatially-varying infinitesimal absorption perturbation is, at a given time (Arridge 1995)

$$
\delta_{\mu_{a}(\mathbf{r})} h_{m, n}(t)=\int \delta \mu_{a}\left(\mathbf{r}^{\prime}, t\right) u_{m}\left(\mathbf{r}^{\prime}, t\right) \tilde{u}_{n}\left(\mathbf{r}^{\prime}, t\right) \mathrm{d}^{3} \mathbf{r}^{\prime}
$$


where $u_{m}(\mathbf{r}, t)$ is the forward solution due to source $m, \tilde{u}_{n}(\mathbf{r}, t)$ is the adjoint solution due to detector $n$, and $\delta \mu_{a}(\mathbf{r})$ is a small functional perturbation in absorption. Using the chain rule, the sensitivity with respect to $p(\mathbf{r})$ of a given amplitude measurement is then

$\delta_{p(\mathbf{r})} h_{m, n}(t)=\int \frac{a\left(\mathbf{r}^{\prime}, t\right)}{\sqrt{a\left(\mathbf{r}^{\prime}, t\right)^{2}+\epsilon}} \delta p\left(\mathbf{r}^{\prime}\right) u_{m}\left(\mathbf{p}, \mathbf{c}, \mathbf{e}, \mathbf{r}^{\prime}, t\right) \tilde{u}_{n}\left(\mathbf{p}, \mathbf{c}, \mathbf{e}, \mathbf{r}^{\prime}, t\right) \mathrm{d}^{3} \mathbf{r}^{\prime}$.

Similarly, we see that the sensitivity of a time-varying measurement due to an infinitesimal perturbation in $e_{i}(\mathbf{r})$ is

$\delta_{e_{i}(\mathbf{r})} h_{m, n}(t)=\int \frac{a\left(\mathbf{r}^{\prime}, t\right)}{\sqrt{a\left(\mathbf{r}^{\prime}, t\right)^{2}+\epsilon}} \delta e_{i}\left(\mathbf{r}^{\prime}\right) b_{i}(t) u_{m}\left(\mathbf{p}, \mathbf{c}, \mathbf{e}, \mathbf{r}^{\prime}, t\right) \tilde{u}_{n}\left(\mathbf{p}, \mathbf{c}, \mathbf{e}, \mathbf{r}^{\prime}, t\right) \mathrm{d}^{3} \mathbf{r}^{\prime}$.

Finally, if we denote $z_{m, n}^{q}$ as the ratio between the $q$ th amplitude measurement of source $m$ and detector $n$ and the first

$$
z_{m, n}^{q}=\frac{h_{m, n}^{q}\left(t_{m}^{q}\right)}{h_{m, n}^{1}\left(t_{m}^{1}\right)}
$$

where the dependence on $p(\mathbf{r}), \mathbf{c}$ and $\mathbf{e}$ is taken to be implicit, then the Fréchet derivative of $z_{m, n}^{q}$ with respect to $e_{i}(\mathbf{r})$ is

$$
\delta_{e_{i}(\mathbf{r})} z_{m, n}^{q} \frac{h_{m, n}^{1}\left(t_{m}^{1}\right)\left(\delta_{e_{i}(\mathbf{r})} h_{m, n}^{q}\left(t_{m}^{q}\right)\right)-h_{m, n}^{q}\left(t_{m}^{q}\right)\left(\delta_{e_{i}(\mathbf{r})} h_{m, n}^{1}\left(t_{m}^{1}\right)\right)}{\left(h_{m, n}^{1}\left(t_{m}^{1}\right)\right)^{2}} .
$$

\subsection{Spatial and temporal regularization}

In order to be able to effectively reconstruct $\mathbf{p}$ and e from noisy measurements, we must make use of spatial regularization, as the inverse problem is ill-posed in these parameters. Given the slow temporal variation that we observe in clinical measurements, we have found that $\mathbf{c}$ can be represented using a small number of parameters and thus can be estimated without explicit regularization. If we do have prior information that the system which we are modeling is slowly-varying, it is beneficial to make use of temporal, as well as spatial, regularization. We make use of this approach to regularization in the case where the temporal basis functions are time-shifted versions of the same function, for example cubic splines or Gaussians. Introducing temporal regularization imposes an additional constraint on the spatiotemporal reconstruction, for instance, that it be smooth temporally as well as spatially. This is accomplished by the penalizing the degree of dissimilarity of the temporal basis function coefficients which are adjacent in time. In our spatio-temporal reconstructions, we have chosen the approach of using as many temporal basis functions as is computationally feasible in the reconstruction, and subsequently making use of temporal regularization, along with an L-curve method (Hansen 1992, Hansen and O'Leary 1993) for choosing the temporal regularization parameter, to impose a further degree of temporal smoothness. This approach avoids as much as possible over-smoothing the reconstruction in time, as the level of temporal regularization is chosen not a priori, but from the data itself, by examination of the L-curve.

Thus, we augment the equations (8)-(10) as follows:

$$
\begin{aligned}
& C_{\text {absolute, reg }}=\sum_{m=1}^{M} \sum_{n=1}^{N} \sum_{q=1}^{Q} \frac{\left(y_{m, n}^{q}-h_{m, n}^{q}\left(t_{m}^{q}, \mathbf{p}, \mathbf{c}, \mathbf{e}\right)\right)^{2}}{\left(\sigma_{m, n}^{q}\right)^{2}}+\lambda_{s}^{s}\|\mathbf{L} \mathbf{p}\|_{2}^{2} \\
& C_{\text {absolute,calibrated,reg }}=\sum_{m=1}^{M} \sum_{n=1}^{N} \sum_{q=1}^{Q} \frac{\left(y_{m, n}^{q}-s_{m} d_{n} h_{m, n}^{q}\left(t_{m}^{q}, \mathbf{p}, \mathbf{c}, \mathbf{e}\right)\right)^{2}}{\left(\sigma_{m, n}^{q}\right)^{2}}+\lambda_{s}^{s}\|\mathbf{L} \mathbf{p}\|_{2}^{2}
\end{aligned}
$$




$$
\begin{aligned}
C_{\text {relative }, \mathrm{s}-\text { treg }}= & \sum_{m=1}^{M} \sum_{n=1}^{N} \sum_{q=2}^{Q}\left[\frac{y_{m, n}^{q}}{y_{m, n}^{1}}-\frac{h_{m, n}^{q}\left(t_{m}^{q}, \mathbf{p}, \mathbf{c}, \mathbf{e}\right)}{h_{m, n}^{1}\left(t_{m}^{1}, \mathbf{p}, \mathbf{c}, \mathbf{e}\right)}\right]^{2} \\
& +\sum_{l=1}^{L} \lambda_{s}^{d}\left\|\mathbf{L} \mathbf{e}_{l}\right\|_{2}^{2}+\lambda_{t}^{d} \sum_{l=2}^{L}\left\|\mathbf{e}_{l}-\mathbf{e}_{l-1}\right\|_{2}^{2}
\end{aligned}
$$

where $\lambda_{s}^{s}$ is the spatial regularization parameter for estimation of the static (non-time-varying) parameters, $\lambda_{s}^{d}$ is the spatial regularization parameter for the dynamic parameters, $\lambda_{t}^{d}$ is the temporal regularization parameter, $\mathbf{e}_{l}$ is the discretized version of the image of the $l^{\prime}$ th temporal coefficient, and $\mathbf{L}$ is a regularization operator. In our implementation, $\mathbf{L}$ is the finite-difference discretization of the Laplacian operator.

In order to simplify the presentation, we vectorize the elements of the above cost functions as follows:

$$
\begin{aligned}
& \mathbf{y}=\left[y_{1,1}^{1} y_{1,2}^{1} \cdots y_{M, N}^{1} \cdots y_{M, N}^{Q}\right]^{T} \\
& \mathbf{h}=\left[h_{1,1}^{1} h_{1,2}^{1} \cdots h_{M, N}^{1} \cdots h_{M, N}^{Q}\right]^{T} \\
& \mathbf{h}^{q}=\left[h_{1,1}^{q} h_{1,2}^{q} \cdots h_{M, N}^{q} \cdots h_{M, N}^{q}\right]^{T} \\
& \mathbf{z}=\left[z_{1,1}^{2} z_{1,2}^{2} \cdots z_{M, N}^{2} \cdots z_{M, N}^{Q}\right]^{T} \\
& \mathbf{f}=\left[f_{1,1}^{2} f_{1,2}^{2} \cdots f_{M, N}^{2} \cdots f_{M, N}^{Q}\right]^{T}
\end{aligned}
$$

where

$$
f_{m, n}^{q}=\frac{y_{m, n}^{q}}{y_{m, n}^{1}} .
$$

We also define the vector $\mathbf{b}_{l}$ which gives the value of basis function $l$ for each measurement:

$$
\mathbf{b}_{l}=\left[b_{l}\left(t_{1}^{1}\right) b_{l}\left(t_{1}^{1}\right) \cdots b_{l}\left(t_{M}^{Q}\right) b_{l}\left(t_{M}^{Q}\right)\right]^{T} .
$$

The vector $\mathbf{b}_{l}^{q}$ gives the value of the basis function $l$ for each measurement in cycle $q$ :

$$
\mathbf{b}_{l}^{q}=\left[b_{l}\left(t_{1}^{q}\right) b_{l}\left(t_{1}^{q}\right) \cdots b_{l}\left(t_{M}^{q}\right) b_{l}\left(t_{M}^{q}\right)\right]^{T} .
$$

Thus, equations (16)-(18) can be rewritten more compactly as follows:

$$
C_{\text {absolute, reg }}=(\mathbf{y}-\mathbf{h})^{T} \boldsymbol{\Sigma}_{n}^{-1}(\mathbf{y}-\mathbf{h})+\lambda_{s}^{s}\|\mathbf{L} \mathbf{p}\|_{2}^{2}
$$

where $\Sigma_{n}$ is the noise covariance matrix, which we will assume to be diagonal in our clinical and simulation studies.

$C_{\text {absolute,calibrated,reg }}=(\mathbf{y}-\operatorname{diag}(\mathbf{s} \otimes \mathbf{d}) \mathbf{h})^{T} \boldsymbol{\Sigma}_{n}^{-1}(\mathbf{y}-\operatorname{diag}(\mathbf{s} \otimes \mathbf{d}) \mathbf{h})+\lambda_{s}^{s}\|\mathbf{L} \mathbf{p}\|_{2}^{2}$

where $\mathbf{s}=\left[s_{1} s_{2} \cdots s_{M}\right]^{T}$ and $\mathbf{d}=\left[d_{1} d_{2} \cdots d_{N}\right]^{T}$.

$$
C_{\text {relative,reg }}=(\mathbf{f}-\mathbf{z})^{T}(\mathbf{f}-\mathbf{z})+\sum_{l=1}^{L} \lambda_{s}^{d}\left\|\mathbf{L} \mathbf{e}_{l}\right\|_{2}^{2}+\lambda_{t}^{d} \sum_{l=2}^{L}\left\|\mathbf{e}_{l}-\mathbf{e}_{l-1}\right\|_{2}^{2} .
$$




\subsection{Inverse problem solution}

In the image reconstruction, we make use of Newton-based nonlinear optimization algorithms to estimate $\mathbf{c}, \mathbf{p}$ and e. We assume that $\mu_{a}^{0}$ is known a priori. As discussed in the previous section, we make use of both absolute and relative cost functions in order to reconstruct the static and dynamic components of the absorption, respectively. Since the values of the two cost functions are not comparable, we reconstruct the static and dynamic images separately, although one could contemplate scaling the cost functions and reconstructing all spatiallyvarying parameters in a single step. We begin by estimating the bulk temporal variation, c, using difference imaging. As we are estimating in this step a small number of parameters, regularization does not seem to be necessary. Next, we proceed to estimate $\mathbf{p}$ and e separately, using absolute and relative cost functions, respectively. The order in which we estimate $\mathbf{p}$ and $\mathbf{e}$ is not extremely significant; in our experimental work, we have seen that these parameters largely decouple from one another, especially when using a combination of absolute and difference imaging. However, accurate estimation of $\mathbf{c}$ seems to be very important in order to attain reasonable results for imaging of both the space-varying but static component of the absorption and the dynamics.

Each iteration of the optimization algorithms is composed of two steps: in the first step, we compute the search direction, using a linearization about the current parameter estimate, and, in the second, a line-search procedure is used to calculate the magnitude of the step to take in the search direction. As the computation of the search direction involves the solution of a very large linear system for practical three-dimensional problems, an iterative algorithm is employed. The general outline of our estimation procedure is as follows:

(i) Estimate c, the bulk temporal variation, using a relative cost function.

(ii) Estimate p, the static absorption image, using an absolute cost function, and given the value of $\mathbf{c}$ estimated in step 1.

(iii) Estimate e, the space-varying and time-varying absorption, using a relative cost function (difference imaging) given the estimates of $\mathbf{c}$ and $\mathbf{p}$ obtained in steps 1 and 2 of the algorithm, respectively.

Specifically, we use the following procedure, in which we have a Newton-based optimization procedure as the 'inner loop' of each step.

(i) Estimate c using equations (10) by iterating the following:

$$
\mathbf{c}_{i+1}=\arg \min _{\alpha} C_{\text {relative }}\left(\mathbf{c}_{i}-\alpha \mathbf{w}_{\mathbf{c}, i}\right)
$$

where

$$
\mathbf{w}_{\mathbf{c}, i}=\left(\mathbf{J}_{\mathbf{c}, i}^{T} \mathbf{J}_{\mathbf{c}, i}\right)^{-1} \mathbf{J}_{\mathbf{c}, i}^{T}\left(\mathbf{f}-\mathbf{z}\left(\mathbf{c}_{i}, \mathbf{p}=0, \mathbf{e}=0\right)\right) .
$$

(ii) Estimate $\mathbf{p}$ using equation (27) or (28) and given $\hat{\mathbf{c}}$, the last iterate of $\mathbf{c}_{i}$ from the first step of the optimization algorithm:

$$
\mathbf{p}_{i+1}=\arg \min _{\alpha} C_{\text {absolute, reg }}\left(\mathbf{p}_{i}-\alpha \mathbf{w}_{\mathbf{p}, i}\right)
$$

where

$$
\mathbf{w}_{\mathbf{p}, i}=\left(\mathbf{J}_{\mathbf{p}, i}^{T} \boldsymbol{\Sigma}_{n}^{-1} \mathbf{J}_{\mathbf{p}, i}+\lambda_{s}^{s} \mathbf{L}^{T} \mathbf{L}\right)^{-1} \mathbf{J}_{\mathbf{p}, i}^{T} \boldsymbol{\Sigma}_{n}^{-1}\left(\mathbf{y}-\mathbf{h}\left(\mathbf{p}_{i}, \hat{\mathbf{c}}, \mathbf{e}=0\right)\right) .
$$

(iii) Estimate e using equation (29) and given $\hat{\mathbf{c}}$ and $\hat{\mathbf{p}}, \hat{\mathbf{p}}$ being the last iterate of $\mathbf{p}_{i}$ estimated during the second step of the optimization algorithm, by iterating

$$
\mathbf{e}_{i+1}=\arg \min _{\alpha} C_{\text {relative }, \mathrm{s}-\text { treg }}\left(\mathbf{e}_{i}-\alpha \mathbf{w}_{\mathbf{e}, i}\right)
$$


where

$\mathbf{w}_{\mathbf{e}, i}=\left(\mathbf{J}_{\mathbf{e}, i}^{T} \mathbf{J}_{\mathbf{e}, i}+\lambda_{s}^{d} \text { block } \operatorname{diag}\left(\mathbf{L}^{T} \mathbf{L}\right)+\lambda_{t}^{d} \mathbf{G}^{T} \mathbf{G}\right)^{-1} \mathbf{J}_{\mathbf{e}, i}^{T}\left(\mathbf{f}-\mathbf{z}\left(\hat{\mathbf{c}}, \hat{\mathbf{p}}, \mathbf{e}_{i}\right)\right)$

and

$$
\mathbf{G}=\left[\begin{array}{ccccc}
-\mathbf{I} & \mathbf{I} & \ldots & & \\
& -\mathbf{I} & \mathbf{I} & \ldots & \\
& & \ldots & & \\
& & \ldots & -\mathbf{I} & \mathbf{I}
\end{array}\right]
$$

In principle, we could iterate this entire procedure, but this would be computationally expensive. We have found in our simulation studies, one of which will be described in detail below, that the above procedure gives accurate results in estimating $a(\mathbf{r})$.

The matrices $\mathbf{J}_{\mathbf{c}}, \mathbf{J}_{\mathbf{p}}$ and $\mathbf{J}_{\mathbf{e}}$ are defined as follows:

$$
\mathbf{J}_{\mathbf{p}}=\left[\begin{array}{c}
\mathbf{J}_{\mathbf{p}}^{1} \\
\mathbf{J}_{\mathbf{p}}^{2} \\
\cdots \\
\mathbf{J}_{\mathbf{p}}^{Q}
\end{array}\right]
$$

where $\mathbf{J}_{\mathbf{p}}^{q}$ is the Jacobian of the measurements made in cycle $q$ with respect to a small pertubation in absorption, each row of which is computed using equation (12). This Jacobian is pre-multiplied by $\operatorname{diag}(\mathbf{s} \otimes \mathbf{d})$ in the case where we are optimizing for the calibration coefficients and $\mathbf{p}$ simultaneously.

$$
\mathbf{J}_{\mathbf{e}}=\left[\begin{array}{cccc}
\mathbf{J}_{\mathbf{e}_{1}}^{2} & \mathbf{J}_{\mathbf{e}_{2}}^{2} & \cdots & \mathbf{J}_{\mathbf{e}_{L}}^{2} \\
\mathbf{J}_{\mathbf{e}_{1}}^{3} & \mathbf{J}_{\mathbf{e}_{2}}^{3} & \cdots & \mathbf{J}_{\mathbf{e}_{L}}^{3} \\
\cdots & \cdots & \cdots & \cdots \\
\mathbf{J}_{\mathbf{e}_{1}}^{Q} & \mathbf{J}_{\mathbf{e}_{2}}^{Q} & \cdots & \mathbf{J}_{\mathbf{e}_{L}}^{Q}
\end{array}\right]
$$

where

$$
\mathbf{J}_{\mathbf{e}_{l}}^{q}=\left(\operatorname{diag}\left(\mathbf{h}^{1}\right)\right)^{-2}\left(\operatorname{diag}\left(\mathbf{h}^{1}\right) \operatorname{diag}\left(\mathbf{b}_{l}^{q}\right) \mathbf{J}_{\mathbf{p}}^{q}-\operatorname{diag}\left(\mathbf{h}^{q}\right) \operatorname{diag}\left(\mathbf{b}_{l}^{1}\right) \mathbf{J}_{\mathbf{p}}^{1}\right)
$$

as can be easily shown using equations (15) and (13). We compute the Jacobian of the measurements with respect to $\mathbf{c}$ as

$$
\mathbf{J}_{\mathbf{c}}=\mathbf{J}_{\mathbf{e}} \mathbf{1}
$$

where $\mathbf{1}$ is a vector of all ones with dimension $(V L) \times 1, V$ being the number of voxels for which we are reconstructing the image.

Computation of the search directions, $\mathbf{u}_{\mathbf{p}, i}$ and $\mathbf{u}_{\mathbf{e}, i}$ for steps 2 and 3 of the optimization algorithm shown above is a computationally intense problem, particularly the computation of $\mathbf{u}_{\mathbf{e}, i}$, which involves the estimation of the spatially-varying perturbations in the images for all of the temporal coefficients from all of the data at once. In order to solve this extremely large linear system, we make use of the LSQR algorithm, which involves the solution of the following equivalent systems in the least-squares sense:

$$
\begin{aligned}
\mathbf{S}_{\mathbf{p}, i} \mathbf{w}_{\mathbf{p}, i} & =\left[\begin{array}{c}
\boldsymbol{\Sigma}_{n}^{-1 / 2}\left(\mathbf{y}-\mathbf{h}\left(\mathbf{p}_{i}, \mathbf{c}, \mathbf{e}=0\right)\right) \\
\mathbf{0}
\end{array}\right] \\
\mathbf{S}_{\mathbf{e}, i} \mathbf{w}_{\mathbf{e}, i} & =\left[\begin{array}{c}
\left(\mathbf{f}-\mathbf{z}\left(\mathbf{e}_{i}, \mathbf{p}, \mathbf{c}\right)\right) \\
\mathbf{0} \\
\mathbf{0} \\
\cdots \\
\mathbf{0}
\end{array}\right]
\end{aligned}
$$


where

$$
\begin{aligned}
\mathbf{S}_{\mathbf{p}, i} & =\left[\begin{array}{c}
\boldsymbol{\Sigma}_{n}^{-1 / 2} \mathbf{J}_{\mathbf{p}, i} \\
\sqrt{\lambda_{s}^{s}} \mathbf{L}
\end{array}\right] \\
\mathbf{S}_{\mathbf{e}, i} & =\left[\begin{array}{cccc}
\sqrt{\lambda_{s}^{d}} \mathbf{L} & \mathbf{0} & \mathbf{J}_{\mathbf{e}, i} & \\
\mathbf{0} & \sqrt{\lambda_{s}^{d}} \mathbf{L} & \ldots & \mathbf{0} \\
\cdots & \cdots & \ldots & \ldots \\
\mathbf{0} & \mathbf{0} & \ldots & \sqrt{\lambda_{s}^{d}} \mathbf{L} \\
-\sqrt{\lambda_{t}^{d}} \mathbf{I} & \sqrt{\lambda_{t}^{d}} \mathbf{I} & \mathbf{0} & \cdots \\
\mathbf{0} & -\sqrt{\lambda_{t}^{d}} \mathbf{I} & \sqrt{\lambda_{t}^{d}} \mathbf{I} & \ldots \\
\cdots & \cdots & \cdots & \ldots \\
\cdots & \mathbf{0} & -\sqrt{\lambda_{t}^{d}} \mathbf{I} & \sqrt{\lambda_{t}^{d}} \mathbf{I}
\end{array}\right] .
\end{aligned}
$$

The dimension of $\mathbf{S}_{\mathbf{p}, i}$ and $\mathbf{S}_{\mathbf{e}, i}$ are $(M N Q+V) \times V$ and $(M N(Q-1)+2 L V) \times L V$, respectively, for the case where $\mathbf{L}$ is the Laplacian matrix.

The LSQR algorithm computes the least-squares solutions for the above systems in an efficient manner, requiring only the computation of $\mathbf{A} \mathbf{u}$ and $\mathbf{A}^{T} \mathbf{v}$, for arbitrary vectors $\mathbf{u}$ and $\mathbf{v}$. The matrices $\mathbf{S}_{\mathbf{p}, i}$ and $\mathbf{S}_{\mathbf{e}, i}$ have a highly sparse structure, particularly as we represent $\mathbf{L}$ as a sparse matrix, allowing these matrix-vector products to be computed with relatively little computation or storage. Additional computational savings can be achieved by noting that $\mathbf{J}_{\mathbf{e}, i}$ itself has a highly redundant structure, as is evident from equation (39).

Finally, we address the question of regularization parameter selection. In the optimization algorithm described above, we make use of three regularization parameters, $\lambda_{s}^{s}, \lambda_{s}^{d}$ and $\lambda_{t}^{d}$. However, as has been previously mentioned, the static and dynamic estimation problems, steps 2 and 3 of the optimization algorithm, which make use of different cost functions, can be largely decoupled from one another. Thus, the selection of $\lambda_{s}^{s}$, the baseline-imaging regularization parameter, can be accomplished by standard methods, such as L-curve analysis (Hansen 1992, 1998, Hansen and O'Leary 1993, Vogel 2002).

The joint selection of $\lambda_{s}^{d}$ and $\lambda_{t}^{d}$, the spatial and temporal dynamic-imaging regularization parameters, respectively, is somewhat more problematic, as we have found that these parameters interact with one another. For the purposes of this work, exploring the full parameter space of $\lambda_{s}^{d}$ and $\lambda_{t}^{d}$ using a technique such as L-surface analysis (Brooks et al 1999, Belge et al 2002) would have been too computationally costly, as producing the full nonlinear spatio-temporal reconstruction for a single $\left\{\lambda_{s}^{d}, \lambda_{t}^{d}\right\}$ pair requires roughly $12 \mathrm{~h}$ of processing time at present. Our purpose in this paper was to demonstrate qualitatively the reduction of noise sensitivity and the increased temporal smoothness of reconstructions produced as we increase the temporal regularization parameter, $\lambda_{t}^{d}$. Thus, we first choose an appropriate level of spatial regularization, $\lambda_{s}^{d}$, using the L-curve, and then, holding the spatial regularization parameter fixed, we select the temporal regularization parameter, $\lambda_{t}^{d}$ using an additional Lcurve analysis. We do anticipate that further improvement in imaging quality may be possible using a method which selects both the spatial and temporal dynamic-imaging regularization parameters simultaneously.

\section{Simulation setup}

Using a finite-difference-based forward model, we have simulated a time-varying breast, using a breast geometry generated from the tomosynthesis reconstruction of a breast cancer 


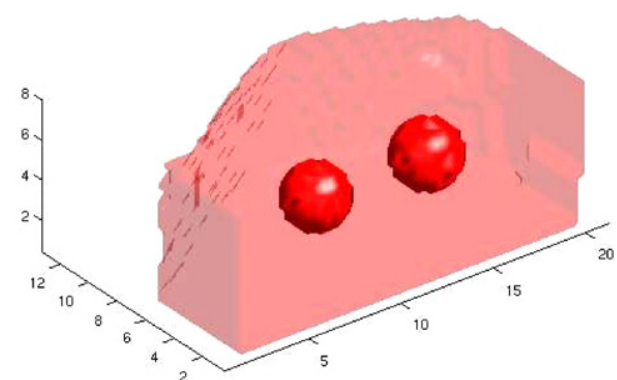

(a)

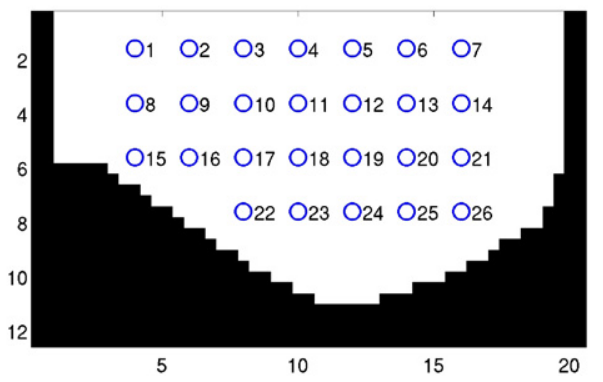

(c)

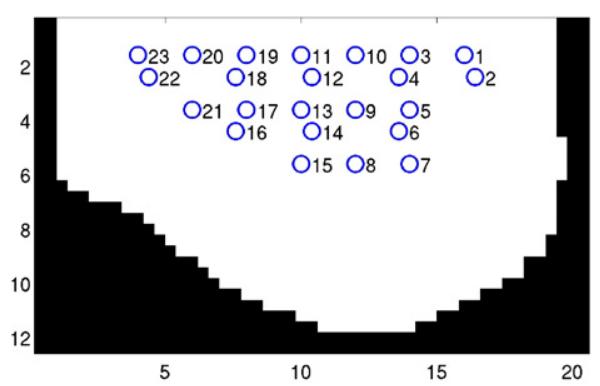

(b)

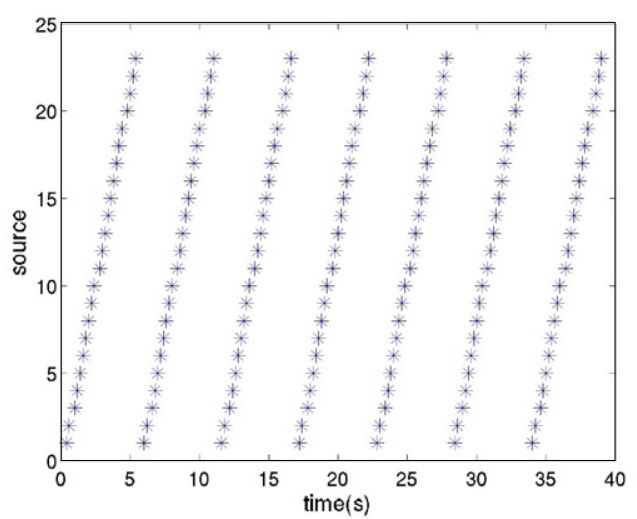

(d)

Figure 2. Simulation setup: (a) geometry used in the simulation, (b) source positions, (c) detector positions and (d) time schedule of sources.

screening subject, age 79. In all images shown, labels on the $x, y$ and $z$ axes of images of the breast denote centimeters. The forward solution was computed with voxels $4 \mathrm{~mm}$ on a side. Figure 2(a) shows the imaging configuration for our simulation study which was based upon the tomosynthesis reconstruction for a breast cancer screening subject, age 79. As shown, we have inserted two inclusions into the geometry. The setup for our clinical experiment was very similar, with only the breast geometry differing. Positions of sources and detectors are shown in figures 2(b) and (c), respectively. The schedule of measurements is depicted in figure 2(d), where the $x$-axis indicates time and the $y$-axis gives the source that is active at each point in time.

We simulate continuous-wave (CW) measurements at 685 and $830 \mathrm{~nm}$ simultaneously, which again corresponds to the wavelengths currently available in our clinical system. Gaussian noise has been added to the simulated measurements such that the SNR of each measurement was $60 \mathrm{~dB}$. In the results which follow, we reconstruct the time-varying absorption at each wavelength separately and, given the measured extinction coefficients of oxy-hemoglobin, deoxy-hemoglobin, water and lipids, convert these estimated absorptions to chromophore concentrations, assuming that the breast is uniformly composed of $35 \%$ water and $35 \%$ lipids (Shah et al 2004). The initial bulk $\mathrm{HbT}$ of the breast was $30 \mu \mathrm{M}$, with $\mathrm{SO}_{2}$ of $70 \%$. All of the reconstructions shown in what follows depict a slice in the center of the breast $(z=2.5 \mathrm{~cm})$.

The simulated breast has been divided into three regions, as shown in figure 2(a), each of which with different temporal and static characteristics. The baseline HbT differs from 


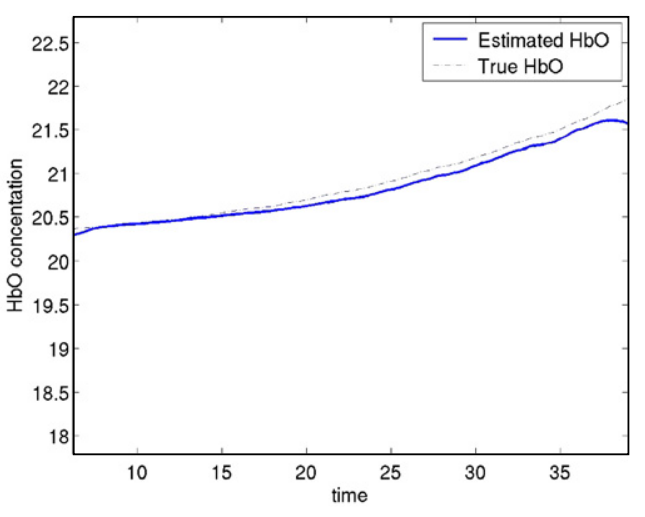

(a)

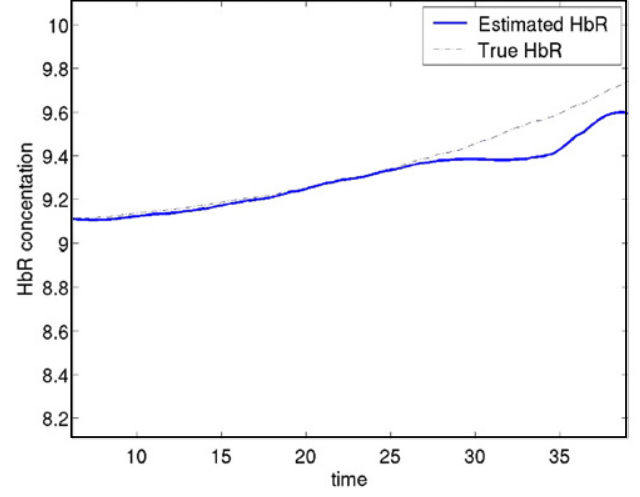

(b)

Figure 3. True and estimated $\mathrm{HbO}$ and $\mathrm{HbR}$ time courses: (a) true and estimated $\mathrm{HbO}$ time courses and (b) true and estimated HbR time courses.

the background for only the left-most object in figure 2(a). The object on the left also experiences the same quadratic increase in $\mathrm{HbT}$ of $1.87 \mu \mathrm{M}$ over the data collection interval as the background, while the object on the right experiences a quadratic decrease in HbT of $1.87 \mu \mathrm{M}$, following the same time course. The $\mathrm{SO}_{2}$ of the background does not vary with time, but the object on the left experiences a decrease in $\mathrm{SO}_{2}$ of $4.4 \%$ over the data collection interval, while the object on the right experiences an increase of $4.4 \%$ with the same time course.

\section{Results and discussion}

\subsection{Simulation study}

The reconstruction of the bulk time-variation in the breast, corresponding to step 1 of our optimization procedure (the estimation of c) is shown in figures 3(a) and (b), which compare the true and reconstructed time courses for oxy-hemoglobin $(\mathrm{HbO})$ and deoxy-hemoglobin $(\mathrm{HbR})$, respectively. We see that the reconstructed and true time courses agree quite well, with somewhat more error in the deoxy-hemoglobin time course. Next, we consider the estimation of $\mathbf{p}$ given $\mathbf{c}$, the estimation of the static (temporally homogeneous) portion of the hemoglobin distribution given the bulk hemodynamics, step 2 of the optimization procedure. In our simulation, the left-most object in figure 2(a) has a greater baseline HbT than the background of the breast, while the object on the right has the same baseline properties as the breast background. If we take into account the estimate of the background time-variation in the breast (c) and reconstruct $\mathbf{p}$ for each wavelength, the images shown in figures 4(a) and (c) are obtained for $\mathrm{HbO}$ and $\mathrm{HbR}$, respectively. The corresponding true images for $\mathrm{HbO}$ and $\mathrm{HbR}$ are shown in figures 4(b) and (d). We see that the background $\mathrm{HbO}$ and $\mathrm{HbR}$ concentrations localize the inclusion very well and quantitatively estimate the increase in $\mathrm{HbO}$ and $\mathrm{HbR}$ concentrations well, with some degree of underestimation due to regularization.

If the medium is truly time-varying, it is quite crucial to take this temporal variation into account when performing the image reconstruction. The effect of ignoring temporal variation in the image reconstruction is illustrated in figure 5, which has the same layout as figure 4. When we ignore the background temporal variation in absorption (i.e. when we assume that $\mathbf{c}=\mathbf{0}$ ), and using the same level of spatial regularization as was used to produce figure 4, 


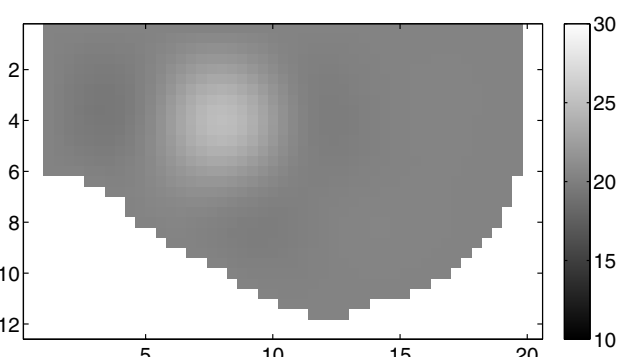

(a)

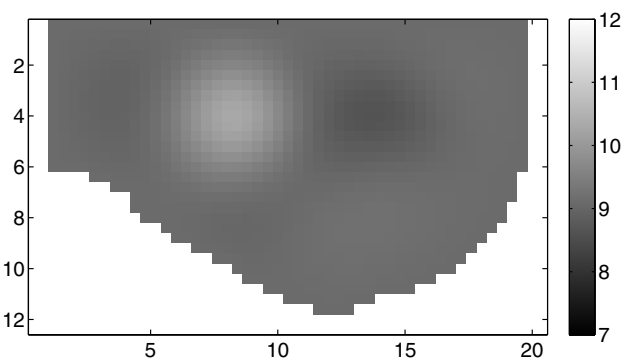

(c)

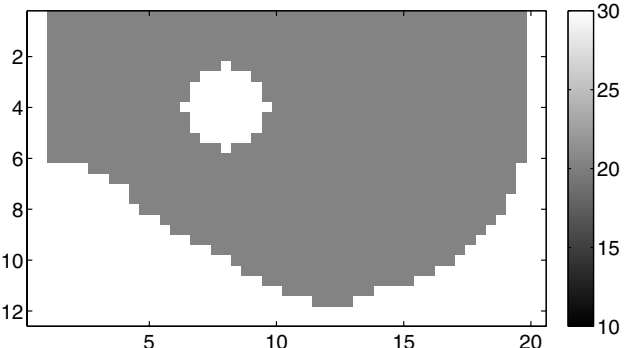

(b)

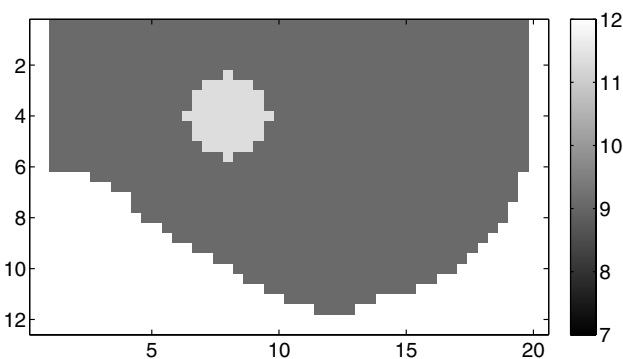

(d)

Figure 4. True and estimated baseline $\mathrm{HbO}$ and $\mathrm{HbR}$ images: (a) estimated $\mathrm{HbO}$ baseline, (b) true $\mathrm{HbO}$ baseline, (c) estimated HbR baseline and (d) true HbR baseline.

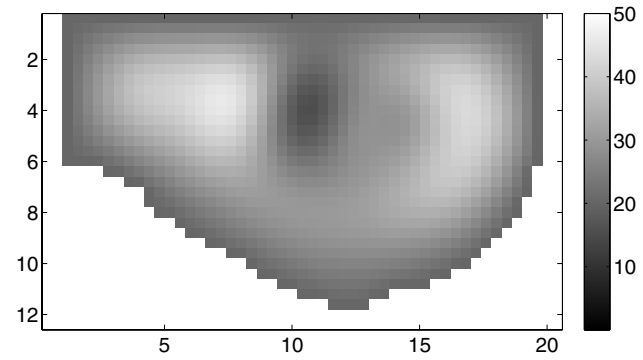

(a)

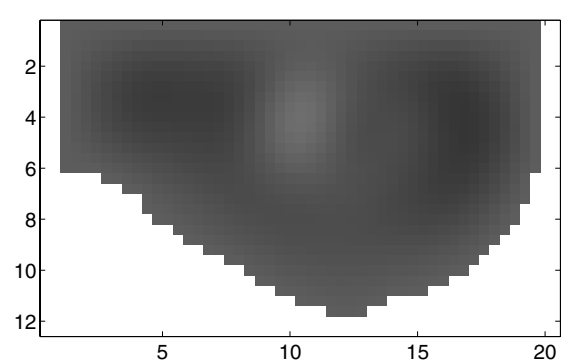

(c)

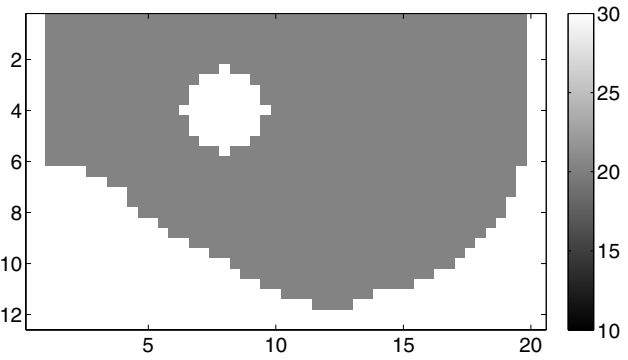

(b)
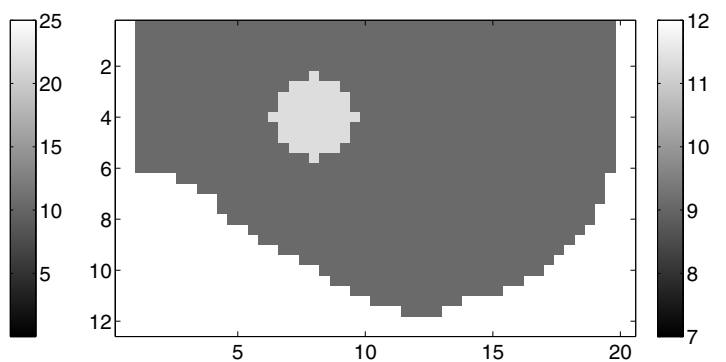

(d)

Figure 5. True and estimated baseline $\mathrm{HbO}$ and $\mathrm{HbR}$ images. Estimated images are reconstructed for the case where it is assumed that there is no bulk temporal variation in optical properties in the breast. (a) Estimated $\mathrm{HbO}$ baseline. (b) True HbO baseline. (c) Estimated HbR baseline. (d) True HbR baseline. 
Table 1. Estimation of HbT as a function of time.

\begin{tabular}{|c|c|c|c|c|c|c|}
\hline & $8.4 \mathrm{~s}$ & $14 \mathrm{~s}$ & $19.6 \mathrm{~s}$ & $25.2 \mathrm{~s}$ & $30.8 \mathrm{~s}$ & $36.4 \mathrm{~s}$ \\
\hline & \multicolumn{6}{|c|}{ Maximum change estimated in $\mathrm{HbT}(\mu \mathrm{M})$} \\
\hline Background & 0.71 & 0.87 & 1.10 & 1.32 & 1.62 & 2.11 \\
\hline Left inclusion & 0.50 & 0.59 & 0.82 & 1.17 & 1.50 & 1.97 \\
\hline \multirow[t]{2}{*}{ Right inclusion } & 0.29 & 0.48 & 0.50 & 0.58 & 0.80 & 1.38 \\
\hline & \multicolumn{6}{|c|}{ True change seen in $\mathrm{HbT}(\mu \mathrm{M})$} \\
\hline Background & 0.14 & 0.30 & 0.54 & 0.87 & 1.31 & 1.87 \\
\hline Left inclusion & 0.14 & 0.30 & 0.54 & 0.87 & 1.31 & 1.87 \\
\hline Right inclusion & -0.14 & -0.30 & -0.54 & -0.97 & -1.31 & -1.87 \\
\hline
\end{tabular}

Table 2. Estimation of $\mathrm{SO}_{2}$ as a function of time.

\begin{tabular}{|c|c|c|c|c|c|c|}
\hline & $8.4 \mathrm{~s}$ & $14 \mathrm{~s}$ & $19.6 \mathrm{~s}$ & $25.2 \mathrm{~s}$ & $30.8 \mathrm{~s}$ & $36.4 \mathrm{~s}$ \\
\hline & \multicolumn{6}{|c|}{ Maximum change estimated in $\mathrm{SO}_{2}(\%)$} \\
\hline Background & -1.1 & 1.0 & 1.3 & 1.3 & 1.4 & 1.3 \\
\hline Left inclusion & 0.4 & -0.4 & -0.7 & -0.9 & -0.8 & -0.7 \\
\hline \multirow[t]{2}{*}{ Right inclusion } & 0.8 & 1.0 & 1.2 & 1.3 & 1.5 & 1.5 \\
\hline & \multicolumn{6}{|c|}{ True change seen in $\mathrm{SO}_{2}(\%)$} \\
\hline Background & 0.0 & 0.0 & 0.0 & 0.0 & 0.0 & 0.0 \\
\hline Left inclusion & -0.3 & -0.7 & -1.3 & -2.1 & -3.1 & -4.4 \\
\hline Right inclusion & 0.3 & 0.7 & 1.3 & 2.1 & 3.1 & 4.4 \\
\hline
\end{tabular}

we see that the visibility of the inclusion is significantly reduced by image artifacts. Thus, in imaging the baseline (non-time-varying) component of our images, it may be of importance to take into account tissue dynamics that take place in the course of our imaging procedure.

Next, we consider the estimation of e given $\mathbf{c}$ and $\mathbf{p}$. The true images of $\mathrm{HbT}$ and $\mathrm{SO}_{2}$, as a function of time, are shown in figures 6(a) and (b). In the simulation, the right-most object in figure 2(a) experiences a decrease in HbT over time, relative to the background, while the object on the left varies in HbT at the same rate as the background. In contrast, both of the inclusions experience changes in $\mathrm{SO}_{2}$ relative to the background, with the left-most inclusion decreasing over time and the right-most inclusion increasing over time. The reconstructed images of $\mathrm{HbT}$ and $\mathrm{SO}_{2}$, using a temporal regularization parameter of $\lambda_{t}^{d}=1.0$, are given in figures 6(c) and (d), respectively. All of the reconstructed images which we will show in the remainder of this paper are differential images, showing only the difference between each parameter at a given time and the value of the parameter at $t=0$.

A quantitative analysis of reconstruction accuracy for $\mathrm{HbT}$ and $\mathrm{SO}_{2}$ has been performed, with the results shown in tables 1 and 2. Each table gives the maximum change from each parameter's value at time $t=0$ as well as the true change, for the three regions of interest: the left inclusion, the right inclusion and the background of the breast. The analysis is given for the six time points corresponding to the images in figure 6. We see that, for HbT, the left inclusion and breast background track the true changes closely, while, in our reconstruction for the right inclusion, HbT increases at a slower rate than the rest of the breast, by $1.38 \mu \mathrm{M}$ as opposed to approximately $2 \mu \mathrm{M}$ in the background and in the left inclusion, when, in reality a decrease of $1.87 \mu \mathrm{M}$ occurred. This phenomenon is a common feature of diffusive imaging, as the spatial regularization tends to reconstruct changes over a larger volume than that in which they actually occurred, with a correspondingly smaller contrast. The quantitative analysis for $\mathrm{SO}_{2}$ is shown in table 2. The relative noisiness of the $\mathrm{SO}_{2}$ is evident 


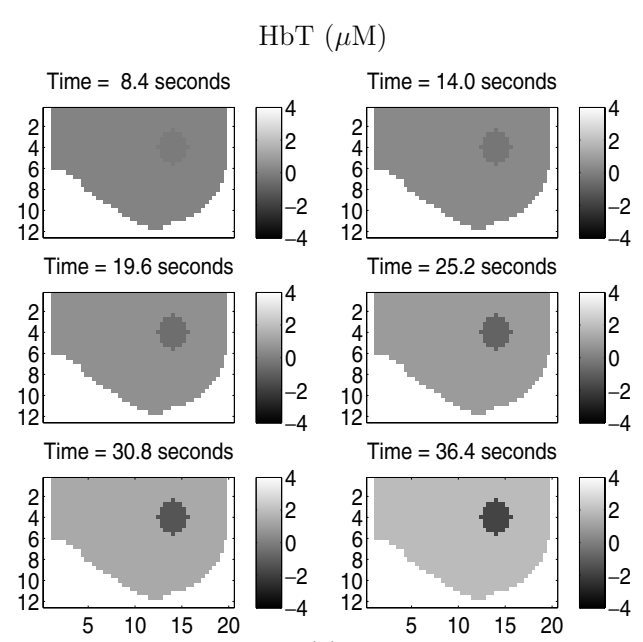

(a)

$\operatorname{HbT}(\mu \mathrm{M})$

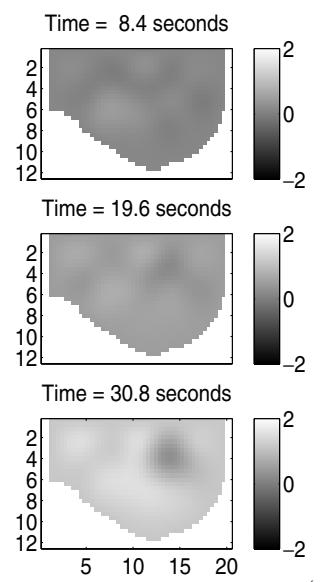

(c)

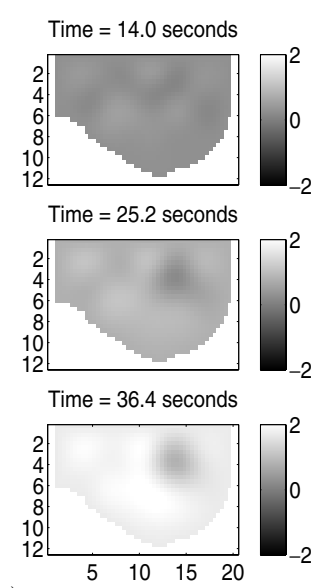

$\mathrm{SO}_{2}$

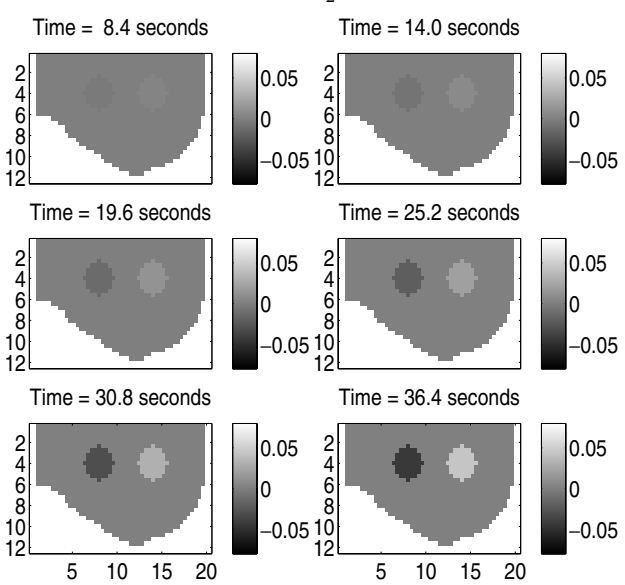

(b)

$\mathrm{SO}_{2}$

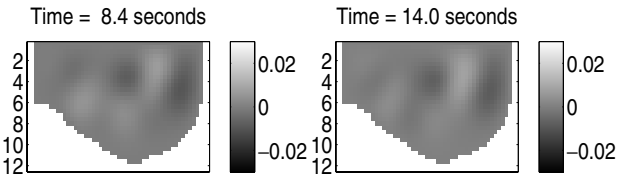

Time $=19.6$ seconds

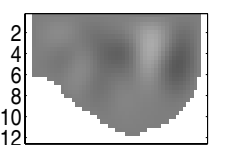

Time $=30.8$ seconds
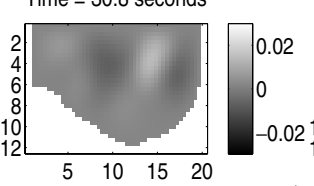

Time $=25.2$ seconds

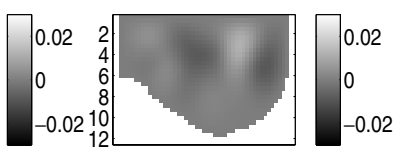

Time $=36.4$ seconds

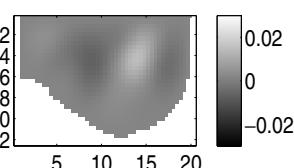

(d)

Figure 6. True and estimated hemodynamic images, where, in the estimated images, a temporal regularization parameter of $\lambda_{t}^{d}=1.0$ was used. (a) True HbT images. (b) True $\mathrm{SO}_{2}$ images. (c) Estimated HbT images. (d) Estimated $\mathrm{SO}_{2}$ images.

in noticing that a change in oxygen saturation of as much as $1.4 \%$ is seen in the background of the breast, when no change should have been seen. The reconstruction correctly finds the left inclusion to be decreasing in oxygen saturation with time and the right inclusion to be increasing, but the magnitudes of the changes are underestimated by $84 \%$ and $66 \%$ for the left and right inclusions, respectively.

\subsection{Pilot clinical study}

Finally, we apply the methods that we have developed and tested for simulated measurements to data acquired from a breast cancer screening subject, age 37. Informed consent was obtained, under Massachusetts General Hospital Institutional Research Board (IRB) protocol no. 1999-P-010998. The experimental protocol was as follows: the patient's breast was compressed in the medio-lateral oblique (MLO) orientation, using a standard mammographic 

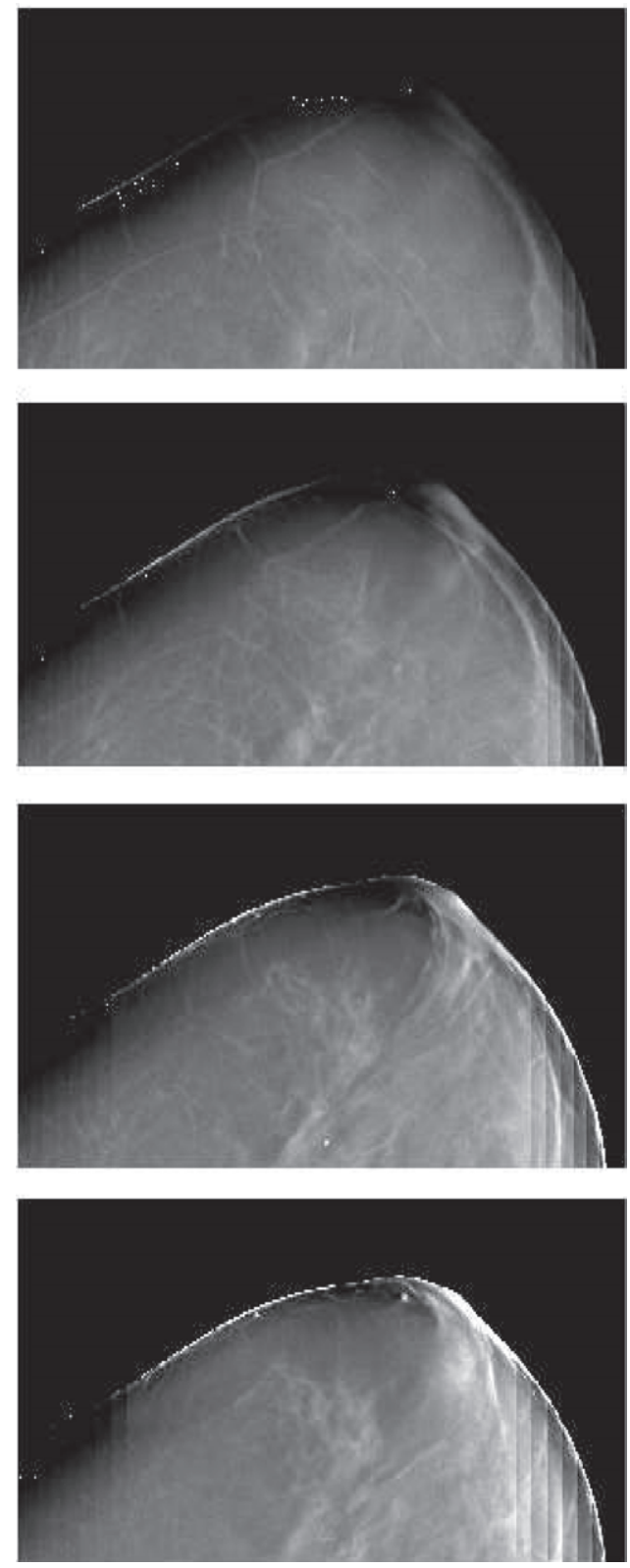

Figure 7. Tomosynthesis reconstruction of a clinical screening subject.

level of compression, and optical data, using both the CW and RF systems, were acquired for approximately $45 \mathrm{~s}$. After acquiring the optical data, the optical probe was removed from its casing, altering the breast position as little as possible, and an x-ray tomosynthesis image of the breast was taken. For the $\mathrm{CW}$ imaging system, a pattern of 28 source positions, with each source turned on for $200 \mathrm{~ms}$, and measurements simultaneously made at $685 \mathrm{~nm}$ and $830 \mathrm{~nm}$, was repeated seven times. Slices of the tomosynthesis reconstruction can be seen in figure 7 . The reconstructions of the bulk time-variation within the breast, for $\mathrm{HbO}$ and $\mathrm{HbR}$, can be seen 


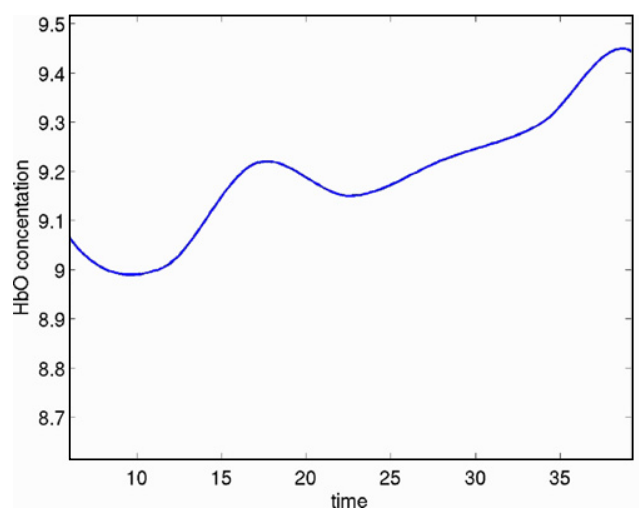

(a)

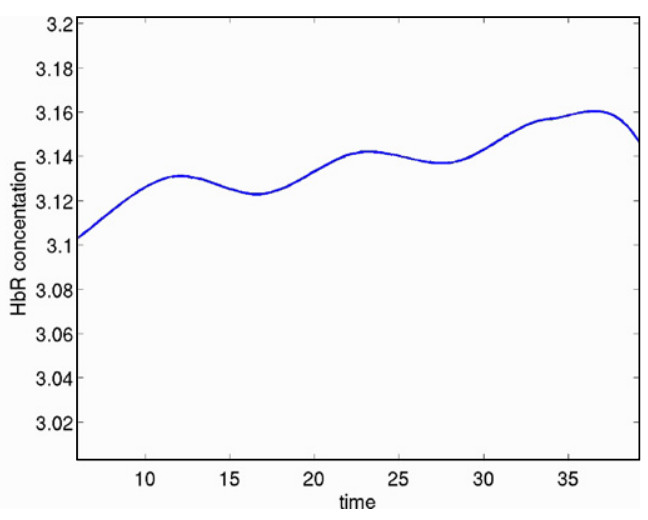

(b)

Figure 8. Estimated background $\mathrm{HbO}$ and $\mathrm{HbR}$ time courses for clinical measurements: (a) estimated $\mathrm{HbO}$ time course and (b) estimated HbR time course.

$\operatorname{HbT}(\mu \mathrm{M})$

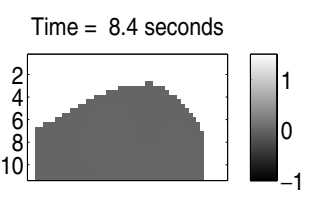

Time $=19.6$ seconds

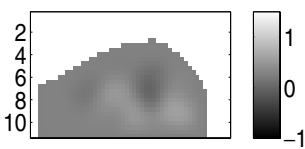

Time $=30.8$ seconds

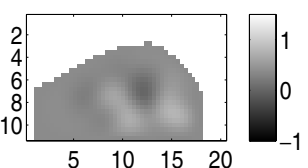

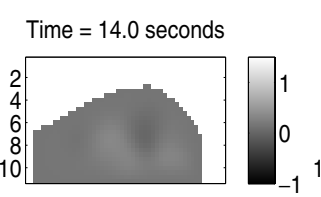

Time $=25.2$ seconds

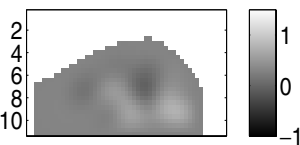

Time $=36.4$ seconds

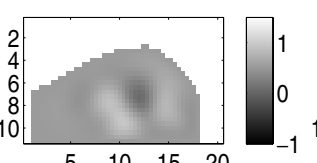

(a)
$\mathrm{SO}_{2}$

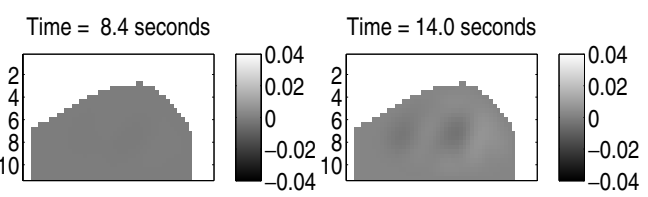

Time $=19.6$ seconds

Time $=25.2$ seconds

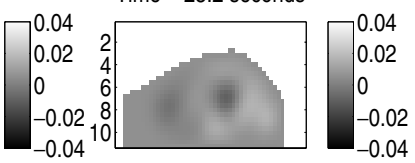

Time $=36.4$ seconds

Time $=30.8$ seconds

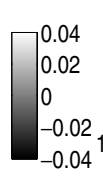

(b)

Figure 9. Estimated images of $\mathrm{HbT}$, and $\mathrm{SO}_{2}$, as a function of time, for the clinical screening subject. (a) $\mathrm{HbT}$ reconstruction. (b) $\mathrm{SO}_{2}$ reconstruction.

in figure 8 . We hypothesize that the return of blood to the breast is induced by deformations of tissue which tend to cause a reduction in pressure during the compression interval. We note that most of the increase in blood within the breast as a function of time is due to $\mathrm{HbO}$, and we hypothesize that perhaps the arterioles exhibit a greater degree of sensitivity to changes in pressure. The oscillatory nature of the time courses is most likely due to the basis functions which we have used, rather than being representative of dynamics that were actually present in the breast. Although we have chosen to make use of the same set of temporal basis functions for the reconstruction of $\mathbf{c}$ as for the reconstruction of $\mathbf{e}$, there is no paricular reason why this must be the case.

We show $\mathrm{HbT}$ and $\mathrm{SO}_{2}$, calculated from reconstruction of $\mathbf{e}$, in figure 9 . We see that the region of glandular tissue in the center of the breast experiences less increase in HbT as a function of time, perhaps due to the increased stiffness of glandular tissue relative to adipose 
tissue, and, in addition, this region experiences a greater decrease in $\mathrm{SO}_{2}$ than surrounding tissue, perhaps due to the increased metabolic activity within glandular tissue. One challenge in the analysis of data from compression measurements is the difficulty of disambiguating effects due to changes in pressure from effects due to metabolic activity or to changes in metabolic activity. The ability to measure blood flow could aid considerably in our ability to disentangle the effects of these two processes, but perhaps modeling-based approaches could be effective to this end as well.

\section{Conclusion and future work}

We have developed a practical algorithm for the fully nonlinear reconstruction of slowlyvarying dynamic DOT measurements, using a combination of absolute and differential measurements, estimating the static component of the image using an absolute cost function, and making use of a differential cost function for estimation of the dynamics. We assume that the time variation within the medium can be decomposed into a combination of temporal basis functions, introducing a certain degree of smoothness and making the time-space image reconstruction problem computationally tractable. Further temporal smoothing is introduced by the use of temporal regularization. Simulation results have shown the possibility of quantitative imaging of both the baseline and the hemodynamics, and further we have shown the importance of taking into account the dynamics in estimating the baseline image. However, for difference imaging, accurate estimation of the baseline seems to be far less important in estimation of the dynamics.

In a normal screening subject, we have imaged the hemodynamics which result immediately following a sharp compression of the breast. CW DOT measurements were taken in a configuration which allows for near-perfect co-registration with a three-dimensional $\mathrm{x}$-ray tomosynthesis image of the breast, and we have observed both a global return of oxyhemoglobin to the breast following the compression, as well as more localized changes in total hemoglobin and oxygen saturation, which correspond to a glandular region of the breast.

In the future, we plan to make use of spectroscopic constraints in our dynamic imaging, which should improve quantification of changes in chromophores of interest. We also intend to examine model-based dynamic image-reconstruction methods (Ntuba 2006), which directly take into account the bio-physics of vascular dynamics. As previous studies have shown the difference between breast tumors and normal tissue with respect to vasculature and metabolism, breast compression, which is already a standard component of mammographic breast screening, may prove useful as a contrast mechanism capable of probing these differences. We plan to more thoroughly address the use of multiple regularization penalty functions and the question of how we can select multiple regularization parameters in a computationally efficient way. Finally, we plan to consider the more general case of a medium in which both the absorption and scattering parameters vary as functions of space and time.

\section{Acknowledgments}

GB, DHB and ELM acknowledge the support of CenSSIS, the Center for Subsurface Sensing and Imaging Systems, under the Engineering Research Centers Program of the National Science Foundation (award number EEC-9986821). DAB, RHM, DBP, QF, SC and JS were supported by the National Institutes of Health. 


\section{References}

Arridge S R 1995 Photon measurement density functions: part I. Analytical forms Appl. Opt. 34 7395-409

Barbour R L, Graber H L, Pei Y, Zhong S and Schmitz C H 2001 Optical tomographic imaging of dynamic features of dense-scattering media J. Opt. Soc. Am. A 18 3018-36

Barbour R L, Graber H L, Schmitz C H, Tarantini F, Khoury G, Naar D J, Panetta T F, Lewis T and Pei Y 2003 Time-frequency analysis of functional optical mammographic images Optical Tomography and Spectroscopy of Tissue: V Proc. SPIE 4955 84-95

Belge M, Kilmer M E and Miller E L 2002 Efficient determination of multiple regularization parameters in a generalized L-curve framework Inverse Problems 18 1161-83

Bluestone A Y, Abdoulaev G, Schmitz C H, Barbour R L and Hielscher A H 2001 Three-dimensional optical tomography of hemodynamics in the human head Opt. Exp. $9272-86$

Boas D A, Gaudette T J and Arridge S R 2001 Simultaneous imaging and opcode calibration with diffuse optical tomography Opt. Exp. 8 263-70

Brizel D M, Scully S P, Harrelson J M, Layfield L J, Bean J M, Prosnitz L R and Dewhirst M W 1996 Tumor oxygenation predicts for the likelihood of distant metastases in human soft tissue sarcoma Cancer Res. 56 941-3

Brooks D H, Ahmad G F, MacLeod R S and Maratos G M 1999 Inverse electrocardiography by simultaneous imposition of multiple constraints IEEE Trans. Biomed. Eng. 46 3-18

Carp S A, Kauffman T, Fang Q, Rafferty E A, Moore R H, Kopans D B and Boas D A 2006 Compression induced changes in the physiological state of the breast as observed through frequency domain photon migration $J$. Biomed. Opt. 11064016

Cerussi A E, Berger A J, Bevilacqua F, Shah N, Jakubowski D, Butler J, Holcombe R F and Tromberg B J 2001 Sources of absorption and scattering contrast for near-infrared optical mammography Acad. Radiol. 8 211-8

Dehghani H, Pogue B W, Poplack S P and Paulsen K D 2003 Multiwavelength three-dimensional near-infrared tomography of the breast: initial simulation, phantom, and clinical results Appl. Opt. 42 135-45

Diamond S G, Huppert T J, Kolehmainen V, Franceschini M A, Kaipio J P, Arridge S R and Boas D A 2006 Dynamic physiological modeling for functional diffuse optical tomography Neuroimage 30 88-101

Eppstein M J, Dougherty D E, Troy T L and Sevick-Muraca E M 1999 Biomedical optical tomography using dynamic parameterization and Bayesian conditioning on photon migration measurements Appl. Opt. 38 2138-50

Fishkin J B, Coquoz O, Anderson E R, Brenner M and Tromberg B J 1997 Frequency-domain photon migration measurements of normal and malignant tissue optical properties in a human subject Appl. Opt. 36 10-20

Franceschini M A, Moesta K T, Fantini S, Gaida G, Gratton E, Jess H, Mantulin W W, Seeber M, Schlag P M and Kaschke M 1997 Frequency-domain techniques enhance optical mammography: initial clinical results Proc. Natl Acad. Sci. USA 94 6468-73

Graber H L, Pei Y and Barbour R L 2002 Imaging of spatiotemporal coincident states by DC optical tomography IEEE Trans. Med. Imaging 21 852-66

Hansen P C 1992 Analysis of discrete ill-posed problems by means of the 1-curve SIAM Rev. 34 561-80

Hansen P C 1998 Rank-Deficient and Discrete Ill-Posed Problems (Philadelphia, PA: SIAM Press)

Hansen P C and O'Leary D P 1993 Use of the L-curve in the regularization of discrete ill-posed problems SIAM J. Sci. Comput. 14 1487-503

Haskell R C, Svaasand L O, Tsay T T, Feng T, McAdams M S and Tromberg B J 1994 Boundary conditions for the diffusion equation in radiative transfer J. Opt. Soc. Am. A $112727-41$

Hebden J C, Gibson A, Austin T, Yusof R M, Everdell N, Delpy D T, Arridge S R, Meek J H and Wyatt J S 2004 Imaging changes in blood volume and oxygenation in the newborn infant brain using three-dimensional optical tomography Phys. Med. Biol. 49 1117-30

Intes X, Ripoll J, Chen Y, Nioka S, Yodh A G and Chance B 2003 In vivo continuous-wave optical breast imaging enhanced with Indocyanine Green Med. Phys. 30 1039-47

Kolehmainen V, Prince S, Prince S R and Kaipio J P 2003 State-estimation approach to the nonstationary optical tomography problem J. Opt. Soc. Am. A $20876-89$

Li A, Boverman G, Zhang Y, Brooks D, Miller E L, Kilmer M E, Zhang Q, Hillman E M C and Boas D A 2005 Optimal linear inverse solution with multiple priors in diffuse optical tomography Appl. Opt. 44 1948-56

Li A, Zhang Q, Culver J P, Miller E L and Boas D A 2004 Reconstructing chromosphere concentration images directly by continuous-wave diffuse optical tomography Opt. Lett. 29 256-8

Li A et al 2003 Tomographic optical breast imaging guided by three-dimensional mammography Appl. Opt. 42 5181-90 
McBride T O, Pogue B W, Gerety E D, Poplack S R, Osterberg U L and Paulsen K D 1999 Spectroscopic diffuse optical tomography for the quantitative assessment of hemoglobin concentration and oxygen saturation in breast tissue Appl. Opt. 38 5480-90

Niklason L T et al 1997 Digital tomosynthesis in breast imaging Radiology 205 399-406

Ntuba D T 2006 Reconstructing hemodynamic parameters in diffuse optical tomographic breast imaging, Master's Thesis Northeastern University, Boston, MA

Oh S, Milstein A B, Millane R P, Bouman C A and Webb K J 2002 Source-detector calibration in three-dimensional Bayesian optical diffusion tomography J. Opt. Soc. Am. A 19 1983-93

Paige C C and Saunders M A 1982 LSQR: an algorithm for sparse linear equations and sparse least squares $A C M$ Trans. Math. Softw. 8 43-71

Parker J A, Kenyon R V and Troxel D E 1983 Comparison of interpolating methods for image resampling IEEE Trans. Med. Imaging 2 31-9

Pogue B W, Poplack S P, McBride T O, Wells W A, Osterman K S, Osterberg U L and Paulsen K D 2001 Quantitative hemoglobin tomography with diffuse near-infrared spectroscopy: pilot results in the breast Radiology 218 261-6

Prince S, Kolehmainen V, Kaipio J P, Franceschini M A, Boas D and Arridge S R 2003 Time-series estimation of biological factors in optical diffusion tomography Phys. Med. Biol 48 1491-504

Shah N, Cerussi A, Eker C, Espinoza J, Butler J, Fishkin J, Hornung R and Tromberg B 2001 Noninvasive functional optical spectroscopy of human breast tissue Proc. Natl Acad. Sci. USA 98 4420-5

Shah N, Cerussi A E, Jakubowski D, Hsiang D, Butler J and Tromberg B J 2004 Spatial variations in optical and physiological properties of healthy breast tissue J. Biomed. Opt. 9 534-40

Siegel A M, Marota J J A and Boas D A 1999 Design and evaluation of a continuous-wave diffuse optical tomography system Opt. Exp. 4 287-98

Srinivasan S, Pogue B W, Brooksby B, Jiang S, Dehghani H, Kogel C, Wells W A, Poplack S P and Paulsen K D 2005 Near-infrared characterization of breast tumors in vivo using spectrally-constrained reconstruction Technol. Cancer Res. Treatment 4 513-26

Vogel C R 2002 Computational Methods for Inverse Problem (Philadelphia, PA: SIAM Press)

Zhang Q et al 2005 Coregistered tomographic x-ray and optical breast imaging: initial results J. Biomed. Opt. 10024033

Zhang Y, Brooks D H and Boas D A 2005 A haemodynamic response function model in spatio-temporal diffuse optical tomography Phys. Med. Biol. 50 4625-44

Zhang Y, Ghodrati A and Brooks D H 2005 An analytical comparison of three spatio-temporal regularization methods for dynamic linear inverse problems in a common statistical framework Inverse Problems 21 357-82 